MITSUBISHI ELECTRIC RESEARCH LABORATORIES

http://www.merl.com

\title{
Reduction of the error floor of binary FSK by nonlinear frequency discriminators
}

\author{
Molisch, A.; Petrovic, R.
}

TR2003-41 May 2003

\begin{abstract}
We consider the error floor of binary FSK due to intersymbol interference in time-dispersive mobile radio channels, with a limiter-discriminator-integrator detector. The errors are caused by bursts in the instantaneous frequency. We propose and verify that a nonlinear frequency discriminator can achieve zero error floor for pure FSK by clipping off these bursts. For filtered FSK, the error floor is not completely removed, but strongly reduced - typically by one or two orders of magnitude. The tighter the filtering, the less effective this nonlinearity is. The nonlinear discriminator can be also used in conjunction with adaptive sampling.
\end{abstract}

This work may not be copied or reproduced in whole or in part for any commercial purpose. Permission to copy in whole or in part without payment of fee is granted for nonprofit educational and research purposes provided that all such whole or partial copies include the following: a notice that such copying is by permission of Mitsubishi Electric Research Laboratories, Inc.; an acknowledgment of the authors and individual contributions to the work; and all applicable portions of the copyright notice. Copying, reproduction, or republishing for any other purpose shall require a license with payment of fee to Mitsubishi Electric Research Laboratories, Inc. All rights reserved.

Copyright (C) Mitsubishi Electric Research Laboratories, Inc., 2003

201 Broadway, Cambridge, Massachusetts 02139 



\author{
MERL - A MITSUBISHI ELECTRIC RESEARCH LABORATORY \\ http://www.merl.com
}

\title{
Reduction of the error floor of binary FSK by nonlinear frequency discriminators
}

\author{
Andreas F. Molisch and Rade Petrovic
}

TR-2003-41 May 2003

\begin{abstract}
We consider the error floor of binary FSK due to intersymbol interference in time-dispersive mobile radio channels, with a limiter-discriminator-integrator detector. The errors are caused by bursts in the instantaneous frequency. We propose and verify that a nonlinear frequency discriminator can achieve zero error floor for pure FSK by clipping off these bursts. For filtered FSK, the error floor is not completely removed, but strongly reduced - typically by one or two orders of magnitude. The tighter the filtering, the less effective this nonlinearity is. The nonlinear discriminator can be also used in conjunction with adaptive sampling.
\end{abstract}

This work may not be copied or reproduced in whole or in part for any commercial purpose. Permission to copy in whole or in part without payment of fee is granted for nonprofit educational and research purposes provided that all such whole or partial copies include the following: a notice that such copying is by permission of Mitsubishi Electric Information Technology Center America; an acknowledgment of the authors and individual contributions to the work; and all applicable portions of the copyright notice. Copying, reproduction, or republishing for any other purpose shall require a license with payment of fee to Mitsubishi Electric Information Technology Center America. All rights reserved. 


\section{Publication History:}

1. First printing, TR-2003-41, May 2003 


\title{
Reduction of the error floor of binary FSK by nonlinear frequency
}

\section{discriminators}

\author{
Andreas F. Molisch ${ }^{a}$ and Rade Petrovic ${ }^{b}$ \\ ${ }^{a}$ A. F. Molisch was with AT\&T Labs Research, Middletown, NJ. \\ He is now with the Department of Electroscience, Lund University, Sweden, \\ and Mitsubishi Electric Research Labs, Cambridge, MA. \\ email: Andreas.Molisch@ieee.org \\ ${ }^{b} \mathrm{R}$. Petrovic is with the Center for Wireless Communications, University of Mississippi
}




\begin{abstract}
We consider the error floor of binary FSK due to intersymbol interference in time-dispersive mobile radio channels, with a limiter-discriminator-integrator detector. The errors are caused by bursts in the instantaneous frequency. We propose and verify that a nonlinear frequency discriminator can achieve zero error floor for pure FSK by clipping off these bursts. For filtered FSK, the error floor is not completely removed, but strongly reduced - typically by one or two orders of magnitude. The tighter the filtering, the less effective this nonlinearity is. The nonlinear discriminator can be also used in conjunction with adaptive sampling.
\end{abstract}

\title{
Index Terms
}




\section{INTRODUCTION}

Binary frequency shift keying (FSK), especially minimum shift keying (MSK) and Gauss-filtered MSK (GMSK) are among the most important modulation formats for mobile radio applications. They are not only used in the GSM (Global System for Mobile communications) cellular system, but also in the cordless standard DECT (Digital Enhanced Cordless Telecommunications) and in the HIPERLAN (HIgh PERformance wireless Local Area Networks) standard [1]. Due to the high data rates that are common today, the performance of binary FSK is often not limited by the noise or co-channel interference, but by the intersymbol interference due to the time dispersion of the mobile radio channel. This is especially true for cordless and WLL (wireless local loop) applications, where equalizers are undesireable because of cost reasons. The error rate due to this ISI is known as "error floor".

Because of the great practical importance, the performance of binary FSK with frequency-discriminator detection (FDD) has been given considerable attention in the literature, see e.g. [2], [3], [4], [5], [6] and references therein. All these theoretical investigations were based on the assumption that the receiver performs an ideal FM/AM conversion, i.e. that the output signal of the frequency discriminator is linearly proportional to the frequency of the received signal. In this paper, we will propose and verify that a nonlinear FM/AM conversion, which we will just call nonlinear discriminator in the following, is preferable for reducing or eliminating the error floor. Specifically, we will use a FM/AM converter that is linear in a certain range, and exhibits a cut-off behaviour beyond that threshold.

The paper is organized the following way: in Sec. 2, we will present the system that forms the basis of our considerations, and describe the mathematical techniques used for the evaluation of the error floor. The following section demonstrates that FM clicks due to the intersymbol interference are the reason for the occurence of the error floor, leading directly to the concept of the "clipping" of the clicks. Section 4 gives results for the error floor as a function of the delay spread of the channel, both for fixed and adaptive determination of the sampling instant. Section 5 finally analyzes the performance in a flat-fading environment, and shows that performance in such a channel is almost as good as that of a conventional discriminator. Section 6 gives a summary and conclusions. 


\section{BASIC SYSTEM AND MATHEMATICAL DESCRIPTION}

Figure 1 depicts the system that forms the basis of our analysis. We use here an equivalent baseband representation, so the carrier frequency $f_{c}$ is of no further importance. At the transmitter, a binary data stream frequencymodulates the carrier, the maximum frequency deviation is $\pm f_{d}$, and the modulation index $h_{\text {mod }}$ is defined as $2 f_{d} T$, where $T$ is the bit duration. For the special case $h_{\text {mod }}=0.5$, we have MSK; because of the great practical importance of this modulation index, all of our examples will use it. However, all theoretical considerations are valid for arbitrary modulation index. The signal is then sent through a channel with the impulse response

$$
h(t, \tau)=a_{1}^{c}(t) \exp \left(j \varphi_{1}(t)\right) \delta\left(\tau-\tau_{1}\right)+a_{2}^{c}(t) \exp \left(j \varphi_{2}(t)\right) \delta\left(\tau-\tau_{2}\right)
$$

Here, $a_{i}^{c}$ and $\varphi_{i}^{c}$ are the amplitudes and phase shifts of the two channel echoes of the transmitted delta pulse. Without restriction of generality, we will assume that $\tau_{1}=0$ and denote the maximum excess delay $\tau_{2}-\tau_{1}=\tau_{2}=\tau$; this excess delay is assumed to be constant. We assume that the two echoes exhibit independent fading, i.e. the complex quantities $a_{i}^{c} \exp \left(j \varphi_{i}^{c}\right)$ are random variables. In principle, there is no restriction on the distribution of those variables; in practice (and henceforth in the paper), they will be complex Gaussian variables either with zero mean (for Rayleigh fading) or with nonzero mean (for Rice fading). A change in those quantities happens on the timescale of the inverse Doppler frequency, which is typically $10 \mathrm{~ms}$ or larger. It is thus reasonable to assume that the channel (i.e. the $\left.a_{i}^{c} \exp \left(j \varphi_{i}^{c}\right)\right)$ remains unchanged for several bit durations. At the receiver, the signal is first filtered by a pre-discriminator filter to reduce the noise and adjacent channel interference. For computational purposes, this filter is usually shifted to the transmitter and thus incorporated in the transmitted signal; this is admissible since the channel was assumed to be quasi-stationary. The received signal then undergoes an FM/AM conversion, i.e., the output of the discriminator is

$$
s_{f d}(t)=\Xi[f(t)]
$$

where $f(t)$ is the instantaneous frequency of the received signal. Our investigation refers to the case that the FM/AM conversion is linear in a specific range and exhibits a cut-off behaviour beyond that range, i.e.

$$
\Xi[f(t)]= \begin{cases}q f_{d} & \text { for } f(t)>q f_{d} \\ f(t) & \text { for }-q f_{d}<f(t)<q f_{d} \\ -q f_{d} & \text { for } f(t)<-q f_{d}\end{cases}
$$


As mentioned above, all previous investigations have assumed that $\Xi[f(t)] \propto f(t)$. The output signal of the discriminator is then filtered either by a low-pass filter

$$
r\left(t_{s}\right)=\frac{1}{T} \int_{-\infty}^{\infty} \Xi[f(t)] h_{l f}\left(t_{s}-t\right) d t
$$

or an integrate-and-dump filter

$$
r\left(t_{s}\right)=\frac{1}{T} \int_{t_{s}}^{t_{s}+T_{i}} \Xi[f(t)] d t
$$

where $T_{i}$ is the integration period (usually equal to the symbol length $T$ ), and $t_{s}$ denotes the sampling instant. We will discuss below how this sampling instant can be determined. We then decide that a +1 was sent if $r\left(t_{s}\right)>0$; otherwise, we decide that -1 was sent.

The model used here is sketched in Fig. 1, is quite general, because it allows arbitrary pre- and post-discriminator filters. The major restriction is the use of a two-delay channel model. However, while this model cannot describe all time-dispersive channels [7], it is well established as standard model and has been used very often in the literature for system performance assessments (see e.g. [8], [9], [10], [11] and references therein).

For the computation of the error floor, we use a method that we recently introduced in [12]. In the first step, we write the received signal as

$$
s(t)=a(t) \exp (j \alpha(t))+b(t) \exp (j \beta(t))
$$

where $a(t)$ and $b(t)$ are the amplitudes, and $\alpha(t)$ and $\beta(t)$ the instantaneous phases of the component signals. Note that $a(t)$ and $b(t)$ incorporate the amplitude of the transmitted signal as well as the attenuation by the channel, and can thus be time-variant even though the channel is quasistationary. The instantaneous frequency of the received signal can then be written as

$$
f(t)=\frac{f_{a}(t)+f_{b}(t)}{2}+\frac{\frac{1-c^{2}(t)}{2} \Delta f(t)-\frac{1}{2 \pi} \frac{d c(t)}{d t} \sin (\Delta \varphi(t))}{1+2 c(t) \cos (\Delta \varphi(t))+c^{2}(t)}
$$

where

$$
\begin{aligned}
f_{a}(t) & =\frac{1}{2 \pi} \frac{d \alpha(t)}{d t} \quad f_{b}(t)=\frac{1}{2 \pi} \frac{d \beta(t)}{d t} \\
c(t) & =\frac{b(t)}{a(t)} \quad \Delta f(t)=f_{a}(t)-f_{b}(t) \quad \Delta \varphi(t)=\alpha(t)-\beta(t)
\end{aligned}
$$

Equation 7 shows that the instantaneous frequency consists of two parts, the average frequency of the component signals, and a correction term. The decision variable $r\left(t_{s}\right)$ is computed by inserting (7) into (4) or (5), respectively. 
For certain initial phase differences $\varphi_{0}=\Delta \varphi\left(t_{s}\right)$, the effect of the dispersion is to reverse the sign of the output, which causes a bit error. We define a function $\chi\left(\varphi_{0}\right)$ to be 0 when $f_{\text {out }}$ has the correct sign, and is 1 when $f_{\text {out }}$ has the wrong sign. The variable $\Delta \varphi_{0}$ is then defined as the support of $\chi\left(\varphi_{0}\right)$, i.e. the range of $\varphi_{0}$ where $\chi\left(\varphi_{0}\right)$ is not equal to zero. Of course, $\chi\left(\varphi_{0}\right)$ and $\Delta \varphi_{0}$ depend on $c$.

The average BER is then computed by the following procedure: for each value of $c$ and each bit combination, we compute the values of $\Delta \varphi_{0}$ by solving the equation $r\left(t_{s}\right)=0$ for $\varphi_{0}$. Solving such a nonlinear equation is simple, because the possible range of solutions is restricted to $[0,2 \pi]$ so that no numerical instabilities can occur. Since the initial phases are distributed uniformly (at least for Rayleigh-fading channels), the error probability is

$$
P_{e}(c)=\frac{\Delta \varphi_{0}}{2 \pi}
$$

Of course, there are values of $c$ where no real solutions of $r\left(t_{s}\right)=0$ exist; for these values of $c, P_{e}=0$. The total error probability is then computed by averaging over the pdf of $c, p_{c}(c)$ :

$$
P_{e}=\int P_{e}(c) p d f_{c}(c) d c
$$

The pdf of the ratio of the channel amplitudes $a_{1}^{c} / a_{2}^{c}$ can be easily computed from elementary stochastic principles

$$
p d f_{c}(c)=\int|y| p d f_{a_{1}^{c}}(y) p d f_{a_{2}^{c}}(y c) d y
$$

When we use $N_{c}$ discrete values of $c$ for our computations, the total numerical effort is $N_{c}$ root searches, where each root search might invoke a function that is only given as an integral [in those cases where the integral in (5) or (4) cannot be solved analytically].

\section{FM CLICKS DUE TO CHANNEL-INDUCED ISI}

Let us now consider the reason for the occurence of errors in a time-dispersive environment. Figure 2 shows the instantaneous frequency of MSK for $c=0.9$ for various values of $\varphi_{0}$. We see that for certain values of the the initial phase, there are bursts in the instantaneous frequency that can become quite large. We call those bursts "FM clicks," which can be generally defined as events when the FM signal phasor undergoes an abrupt shift in the phase angle due to noise or interference.The maximum height of such a burst is (for unfiltered modulation)

$$
\xi=\frac{1+c}{1-c} f_{d}
$$


The maximum length of these bursts is $\tau$, so that the maximum contribution of the burst to the frequency integral is $\xi \tau ;$ the contribution of the undisturbed part is $f_{d}(T-\tau)$. If $\xi \tau>f_{d}(T-\tau)$, errors can occur, but the actual occurence of errors also depends on the preceding and the following bits.

When looking at Fig. 2, the idea of limiting the instantaneous frequency to the range $\left[-f_{d}, f_{d}\right]$ becomes obvious. We know that by definition, the transmitted frequencies can lie only in this range, so that anything outside of it must be an artefact of the channel and can be eliminated. More importantly, since now $\xi=f_{d}$, we cannot get errors if $\tau<T / 2$. The "clipping" of the bursts of the instantaneous frequency thus completely eliminates the error floor in a time-dispersive channel with maximum excess delay smaller than half the bit duration. ${ }^{1}$ Furthermore, this technique is exceedingly simple, using no knowledge about the channel, and requiring only a limiter as an additional circuit element in the receiver.

The technique is less effective when transmitter or pre-discriminator receiver filtering is used. Such filtering "smears" the instantaneous frequency bursts over a larger time range - even if $\tau$ is very small, the burst can extend over a whole bit length or more. This can lead to wrong decisions even when the clipping is applied. Figure 3 shows the instantaneous frequency under the same circumstances as Fig. 2, but with Gaussian filtering.

One might now think that clipping the instantaneous frequency bursts by the limiter is equivalent to putting a brickwall filter in front of the discriminator - after all, this should eliminate large frequencies just as well. However, the nonlinear discriminator eliminates large instantaneous frequencies, while the brickwall filter eliminates large spectral frequencies. A brickwall filter always leads to smearing of the symbol transitions and intersymbol interference, while the nonlinear frequency discriminator does not.

\section{ERROR FLOOR OF NONLINEAR DISCRIMINATOR}

\section{A. Clipping with fixed sampling}

Mathematically, the clipping is described by Eq. 3, where in the following we set $q=1$ if not stated otherwise. Inserting this function into the equations of Sec. 2 allows computation of the actual error floor.

${ }^{1}$ The exact amount of the tolerable maximum excess delay depends on the sampling time. If there is no filtering, the instantaneous frequency burst is restricted to the bit transition region. Thus, by choosing the sampling time to be in the middle between the arrival of the first and the last path, we can tolerate even excess delays up to $\tau<T$. 
Figure 4 shows the error floor as a function of the maximum excess delay for various pre-discriminator filter widths. As could be anticipated from the above discussion, the error floor is zero when no filtering is applied, and the output of the frequency discriminator is processed with an integrate-and-dump filter. For finite filtering, the error floor takes on finite values. We see that

$$
P_{e}=K\left(\frac{S}{T}\right)^{2}
$$

where $S$ is the rms delay spread, and the proportionality constant $K$ strongly depends on the filtering; note that this relation is only valid if the channel is Rayleigh-fading with independent paths, and the maximum excess delay is smaller than the bit duration. We see first that the error floor is decreased considerably by using our proposed nonlinear frequency discriminator. However, there is a strong dependence on the used filter bandwidth. For a normalized bandwidth $B_{r} T=0.75$, the error floor is decreased by about a factor of 5 . For $B_{r} T=1.25$, the error floor is decreased by an order of magnitude, and for $B_{r} T=1.75$, it is decreased by two orders of magnitude. The decrease for still larger bandwidths is even stronger; however, using such wideband filters would lead to intolerable adjacent channel interference and noise. Figure 5 shows the error floor for GMSK, with a normalized 3dB filter width of the Gaussian filter of $B_{G} T=0.5$, which corresponds to the DECT specifications. ${ }^{2}$ We see that a complete elimination of the error floor is not possible even if the receiver filter has a very large bandwidth, but still a reduction of the BER by an order of magnitude is feasible for realistic filter parameters.

We also found that a strong reduction of the error floor can be achieved only for binary FSK, as demonstrated above, but not for $M$-ary FSK. We studied the 4-FSK case experimentally in our (Univ. of Mississippi) laboratory. In this case, only the highest and the lowest frequencies can be clipped, which drastically reduces the effectiveness of the scheme.

\section{B. Clipping with adaptive sampling}

Up to now, we have always assumed that the sampling instant $t_{s}$ is at some fixed time, i.e. independent of the instantaneous channel parameters $a_{1}, a_{2}, \varphi_{1}$, and $\varphi_{2}$. As shown in [14], however, the error floor can be reduced considerably when the sampling instant is adjusted according to the channel parameters. The optimum sampling instant is actually found by means of a training sequence; this approach is called adaptive sampling. With

\footnotetext{
${ }^{2}$ Note that in DECT, $T=0.87 \mu \mathrm{s}$.
} 
conventional differential phase detection, the error floor can be completely eliminated for unfiltered MSK. For filtered MSK, the error floor is finite.

The physical mechansism that avoids errors in the adaptive sampling approach is different from the one that is used by the nonlinear discriminator. Roughly speaking, adaptive sampling always chooses the point of maximum eye opening to make decisions - this point is shifted by the group delay of the channel. The nonlinear frequency discriminator, on the other hand, tries to avoid eye closures in the first place. Thus it seems reasonable that the nonlinear discriminator can be gainfully combined with adaptive sampling. Computations can be done most easily by modifying the "error region method" of Ref. [14].

(i) in a first step, we compute the amplitudes and phases of the contributions of the first and second paths, i.e. $a(t), b(t), \alpha(t), \beta(t)$ for all bit combinations of interest. Due to the filtering, up to 7 bits can influence the decision, so that 128 bit combinations must be included.

(ii) next, we describe each channel constellation $\left(a_{1}^{c}, a_{2}^{c}, \varphi_{1}, \varphi_{2}\right)$ by a point in the complex plane, $z=a_{2}^{c} / a_{1}^{c} \exp \left[j\left(\varphi_{2}-\right.\right.$ $\left.\left.\varphi_{1}\right)\right]$. For each such point and each bit combination $\vec{m}, c(t)$ and $\Delta \varphi(t)$ follow immediately, so that $f(t)$ for all times can be computed via Eq. 7.

(iii) integrating $f(t)$ in the interval $\left[t_{s}, t_{s}+T\right]$ for the $i-t h$ possible value of the sampling instant $t_{s}$ gives the output $r(z, \vec{m}, i)$. If this value is larger than 0 , the detector decides that a +1 was transmitted; otherwise the detector decides for -1 . If this decision agrees with the actually transmitted bit, $P e(z, \vec{m}, i)=0$; otherwise $\operatorname{Pe}(z, \vec{m}, i)=1$

(iv) for each channel constellation $z$ and sampling time $i$, the number of wrong decisions is averaged over all bit combinations

$$
P e(z, i)=\frac{1}{2^{N_{b i t}}} \sum_{\vec{m}} P e(z, \vec{m}, i)
$$

(v) the sampling instant that results in the lowest $P e$ for this channel constellation is chosen for the actual transmission:

$$
P e(z)=\min _{i} P e(z, i)
$$

(vi) the error probability is averaged over all possible channel constellations $z$, analoguously to Eq. 10 .

Figure 6 shows the results of the BER computations for a two-delay channel with equal average powers in the two paths and delay spread $S=0.4 T$ for various pre-discriminator filter bandwidths. Results are shown for both 
linear and nonlinear discriminators. If we have no filtering, then the error floor is zero in both cases. In the case of finite filtering, the improvement by the nonlinear discriminator strongly depends on the filter bandwidth. For very strong filtering, $B_{r} T=0.3$, there is practically no improvement. For weak filtering, $B_{r} T=0.75$, the improvement is almost an order of magnitude.

\section{Diversity}

Finally, let us consider whether the nonlinear discriminator can be efficiently combined with diversity. As is well known, the error floor in a two-branch antenna selection diversity arrangement is usually proportional to the fourth power of the normalized delay spread, $B E R=K(S / T)^{4}$ [3], [15]. This is true both for antenna selection that is based on received signal power or a training-sequence based selection; only the proportionality constant is different [15]. We found that this relation is also retained when the nonlinear discriminator is utilized; the actual values of the proportionality constant $K$ depend on the receiver filtering. Since the nonlinear discrimination influences the proportionality constant $K$, and the diversity changes the delay spread dependence from $(S / T)^{2}$ to $(S / T)^{4}$, the combined use of nonlinear discrimination and diversity adds the beneficial effects of these two techniques. This can also be easily shown with computations along the lines of [15]. Results for a filter bandwidth $B_{r} T=0.75$ are shown in Fig. 7.

\section{NOISE PERFORMANCE}

The above results have shown us that the nonlinear discriminator is highly effective for reducing the error floor. For application in actual mobile radio systems, also the behavior in a flat-fading channel is important. From physical considerations, we can anticipate that the performance will be worse than for a linear discriminator. Let us suppose that a +1 is transmitted so that the nominal instantaneous frequency is $+f_{d}$. For a linear discriminator, there are always noise contributions that increase and some that decrease the instantaneous frequency. For large SNR, the deviation from $f_{d}$ is approximately a zero-mean Gaussian random variable [16]. The integration process at the integrate-and-dump filter reduces the variance, but retains the zero-mean Gaussian properties.

When the nonlinear discriminator is applied, then all contributions that increase the instantaneous frequency are cut off, while those that decrease it remain. The deviation from the nominal instantaneous frequency is thus a 
one-sided Gaussian variable, biased towards the value 0 , i.e. towards the decision boundary. Integrating over one bit length over these deviations, the noise contribution is a random variable that has a negative mean value, and thus leads to a larger probability of errors. An exact computation of the BER in the flat-fading channel is rather complicated: the output signal at the sampling instant is the integral of (correlated or uncorrelated) one - sided Gaussian variables. However, it is straightforward to analyze the deterioration of the effective SNR by Monte Carlo simulation. The result of such a simulation with various cut-off levels is shown in Fig. 8. This figure gives results for various levels of SNR, with the delay spread fixed at $S=0.2 T$. At an SNR of $10 d B$, where the BER is dominated by noise, the use of the nonlinear discriminator leads to a slight increase of the BER, namely from 0.05 to 0.07 . At high SNR, the BER is drastically reduced by the nonlinearlty, as expected. We depict not only results where the cutoff is at $f_{d}$, but also at higher levels $q f_{d}$, with $q>1$. The larger the cut-off level, the smaller the increase in the BER (of course, larger cut-off levels lead to a higher error floor). The optimum value of the cutoff parameter depends on the applications in mind. For cordless systems, where SNRs are typically $30 d B$ or larger, a cutoff at $f_{d}$ will usually be best, while narrowband paging systems will be more influenced by noise and thus use a higher cutoff level. A cutoff-level of $2-3$ might be a good compromise if a fixed value has to be implemented a priori.

\section{SUMMARY AND CONCLUSIONS}

In this paper we have proposed and analyzed a novel method for reducing the ISI-induced errors in mobile radio systems that use FSK modulation. This reduction can be achieved by using a nonlinear frequency discriminator, in other words, clipping the instantaneous frequency bursts that are due to intersymbol interference and lead to FM clicks. This has a large impact on the design of receivers for all modulation systems where some form of unequalized FSK, most notably MSK, is used in a time-dispersive fading environment, especially cordless telephones and simulcast systems.

The nonlinear discriminator can be combined with two other schemes for the reduction of the error floor of unequalized systems:

- Diversity can be used in conjunction with the nonlinear discriminator, combining the advantages of these two schemes. 
- Adaptive sampling can be used in conjunction with the nonlinear discriminator. In this case, the error reduction capabilities do not add up fully: the addition of adaptive sampling to the nonlinear discriminator leads only to a reduction of the error floor by a factor on the order of $2-10$. In many receivers, adaptive sampling has to be implemented anyway for synchronization purposes, and its combination with the nonlinear discriminator requires no extra effort.

It is also interesting to compare the nonlinear discriminator with another scheme that reduces the bursts of the instantaneous frequency. Fractional-bit detection [17] eliminates them not by clipping of the instantaneous frequency, but by excluding the bit transition regions from the integration. This has the disadvantages that the maximum excess delay of all possible channels $\tau_{p}$ in which the system will operate must be fixed a priori (either a typical case or a worst case); adaptive determination of the sampling instants and integration length for this case would make for a very complex receiver. Furthermore, a percentage $\tau_{p} / T$ of the arriving signal energy is always thrown away, which increases noise-induced errors. ${ }^{3}$ The scheme is also sensitive to filtering.

On the other hand, the nonlinear discriminator we propose delivers better error floor performance without complicating the receiver structure, and is fully compatible with all existing standards using binary FSK. The only required additional circuit element is a limiter, and even this can usually be implemented in the A/D converter. The price paid is a slight deterioration of the noise performance.

We have also found that filtering reduces the beneficial effect of the nonlinearity: the tighter the receiver (or transmitter) filter, the more the ISI is smeared across the whole duration of the bit and cannot be eliminated by the clipping. On the other hand, too wide a filter bandwidth increases the susceptibility to noise and adjacent channel interference. A good receiver must be designed for the specific purpose at hand. For example, in cordless telephones and WLL (wireless local loop) applications, noise and adjacent channel interference are severe problems; simulcast systems, on the other hand, might require tighter filters for noise reduction.

Acknowledgements: We thank Prof. Dr. Ernst Bonek for valuable discussions and Dr. Juha Laurila for critical reading of the manuscript. Part of this work was supported financially by the Austrian Fonds zur Förderung der wissenschaftlichen Forschung.

\footnotetext{
${ }^{3}$ Note that the case of no post-discriminator filter, which has also been proposed in the literature, can be considered a limiting case of this arrangement.
} 


\section{REFERENCES}

[1] J. Gibson, The mobile radio handbook. IEEE Press, 1996.

[2] P. A. Bello and B. Nelin, "Predetection diversity combining with selectively fading channels," IEEE Trans. Comm., pp. 32-42, 1962.

[3] F. Adachi and J. Parsons, "Error rate performance of digital fm mobile radio with postdetection diversity," IEEE Trans. Comm., vol. 37, pp. 200-210, 1989.

[4] P. Varshney and S. Kumar, "Performance of gmsk in a land mobile radio channel," IEEE Trans. Vehicular Techn., vol. 40, pp. 607-615, 1991.

[5] I. Korn, "Gmsk with limiter discriminator detection in satellite mobile channel," IEEE Trans. Comm., vol. 39, pp. 94-101, 1991.

[6] D. Wedge, "Error rate analysis of broadband binary fm in an indoor channel," in Proc. PIMRC'97, pp. 251-255, 1997.

[7] V. Fung, T. Rappaport, and B. Thoma, "Bit error simulation for pi/4 dqpsk mobile radio communications using two-ray and measurmentbased impulse response models," IEEE Selected Areas Comm., vol. 11, pp. 393-405, 1993.

[8] T. S. Rappaport, Wireless Communications Principles and Practice. IEEE Press, 1996.

[9] I. Korn, "Differential phase shift keying in two-path rayleigh channel with adjacent channel interference," IEEE Trans. Vehicular Techn., vol. 40, pp. 461-471, 1991.

[10] C. L. Liu and K. Feher, "Bit error performance of pi/4-dqpsk in a frequency-selective fast rayleigh fading channel," IEEE Trans. Vehicular Techn., vol. 40, pp. 558-568, 1991.

[11] A. Molisch, J. Fuhl, and P. Proksch, "Error floor of msk modulation in a mobile-radio channel with two independently-fading paths," IEEE Trans. Vehicular Techn., vol. 45, pp. 303-309, 1996.

[12] R. Petrovic and A. F. Molisch, "Multipath effects of fsk with frequency-discriminator detection," IEEE Trans. Vehicular Techn., vol. submitted, 1998.

[13] A. Papoulis, Probability, random variables, and stochastic processes. McGraw Hill, 1965.

[14] A. Molisch, L. Lopes, M. Paier, J. Fuhl, and E. Bonek, "Error floor of unequalized wireless personal communications systems with msk modulation and training-sequence-based adaptive sampling," IEEE Trans. Comm., vol. 45, pp. 554-562, 1997.

[15] A. Molisch, H. Novak, J. Fuhl, and E. Bonek, "Reduction of the error floor of msk by selection diversity," IEEE Trans. Vehicular Techn., vol. in press, 1998.

[16] H. Taub and D. L. Schilling, Principles of Communication Systems. McGraw Hill, 1986.

[17] I. Crohn and E. Bonek, "Suppression of the irreducible errors in a frequency channel by fractional-bit differential detection," IEEE Trans. Vehicular Techn., vol. 43, pp. 1039-1047, 1994. 


\section{Figure captions}

Fig. 1 Block diagram of the analyzed system.

Fig. 2 Instantaneous frequency of unfiltered MSK in a channel with $c=0.9, \varphi_{0}=-0.9 * \pi+i * 0.5 * \pi$, $i=0,1,2,3$.

Fig. 3 Instantaneous frequency of filtered MSK in a channel with $c=0.9, \varphi_{0}=-0.9 * \pi+i * 0.5 * \pi$, $i=0,1,2,3$. Receiver filter with $B_{r} T=3$. Filter impulse response $\exp \left(-B_{r}^{2} t^{2}\right)$.

Fig. 4 Error floor as a function of the delay spread for MSK with nonlinear discriminator reception for various filter bandwidths $B_{r} T$. Filter impulse response $\exp \left(-B_{r}^{2} t^{2}\right)$. Results for linear discriminator shown as dotted lines for comparison. Channel: two independent Rayleigh-fading paths with identical mean average power in the two paths.

Fig. 5 Error floor as a function of the delay spread for GMSK $\left(B_{G} T=0.5\right)$ with nonlinear discriminator reception for various filter bandwidths $B_{r} T$. Receiver filter is a tenth-order Butterworth filter. Channel: two independent Rayleigh-fading paths with identical mean average power in the two paths.

Fig. 6 Error floor of MSK with adaptive sampling with linear (solid) and nonlinear (dashed) discriminator as function of receiver filter bandwidth. Filter impulse response $\exp \left(-B_{r}^{2} t^{2}\right)$. Channel: two independent Rayleighfading paths with identical mean average power in the two paths.; delay spread $S=0.4 T$. Filter transfer function as in Fig. 3.

Fig. 7 Error floor of MSK with nonlinear discriminator with fixed sampling and two-branch diversity reception as function of delay spread. No diversity (solid), RSSI-driven diversity (dashed), and BER-driven diversity (dotted). Filter impulse response $\exp \left(-B_{r}^{2} t^{2}\right), B_{r} T=0.75$.

Fig. 8 BER for various cutoff levels $q f_{d}$. Modulation format: MSK with wideband receiver filter. SNR=10, 20, 30dB, delay spread $S=0.2 T$. 


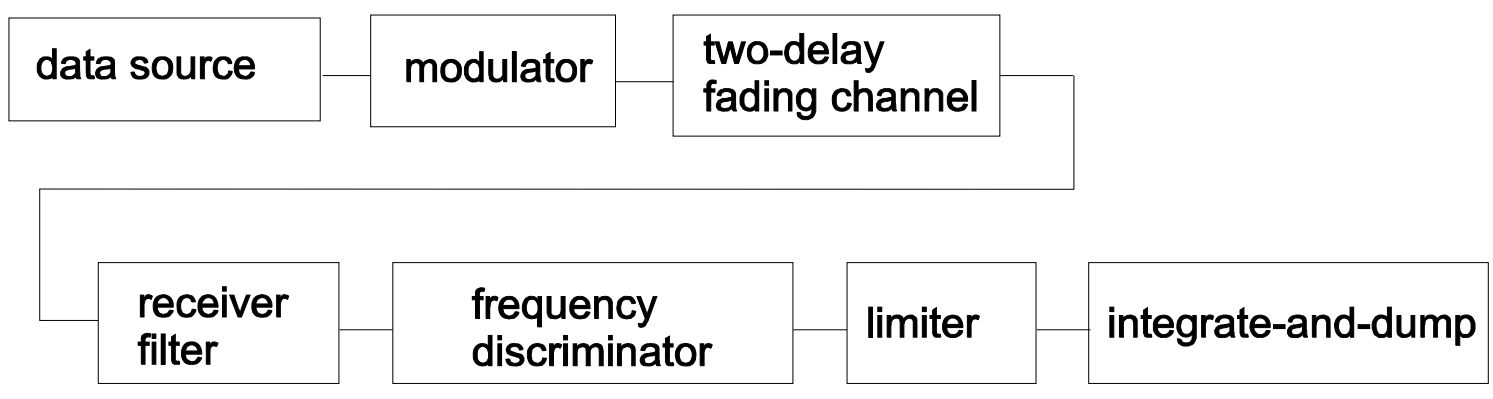

Molisch and Petrovic, Fig. 1

Fig. 1. 


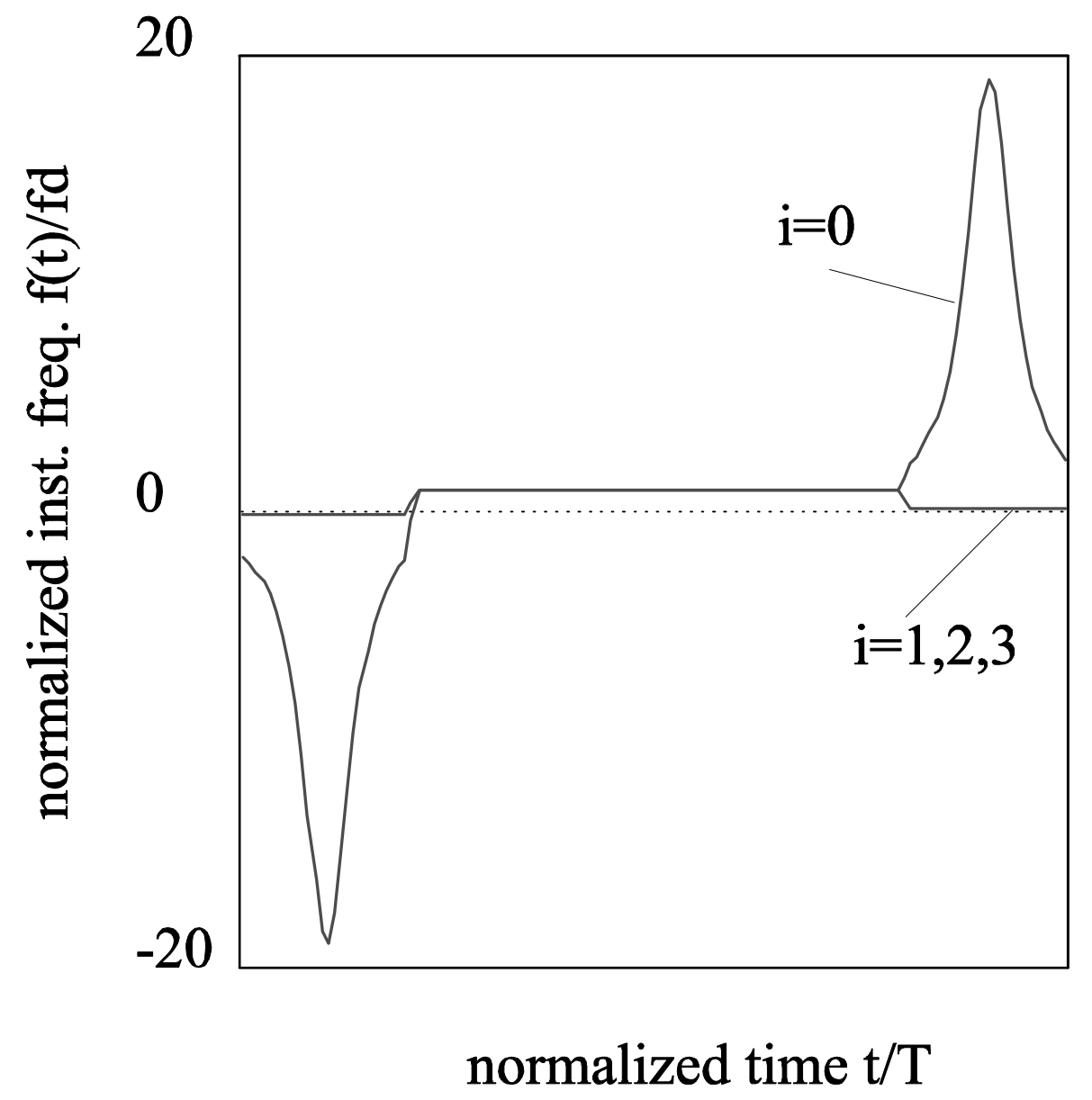

Molisch and Petrovic, Fig. 2

Fig. 2. 


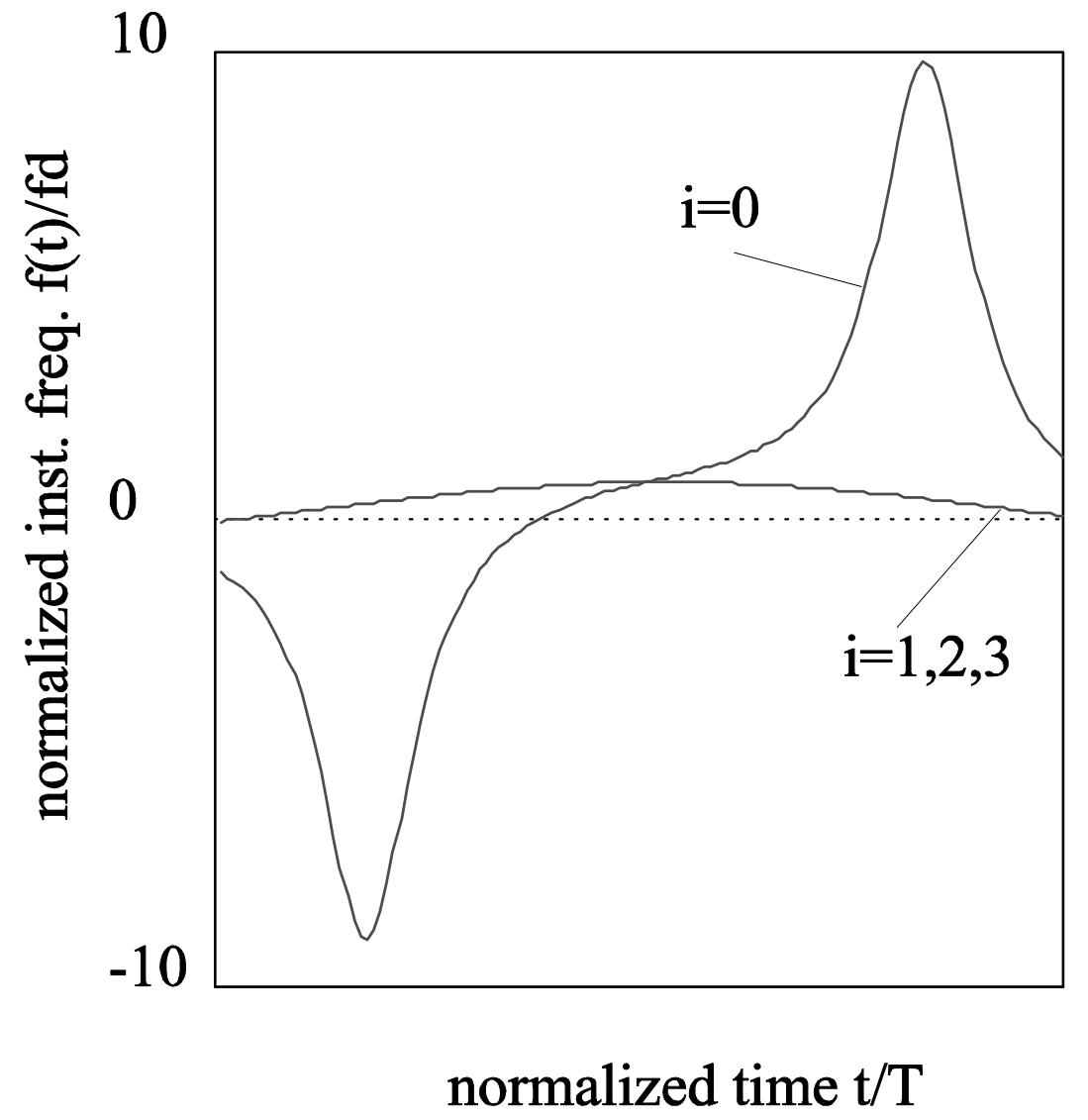

Molisch and Petrovic, Fig. 3

Fig. 3. 


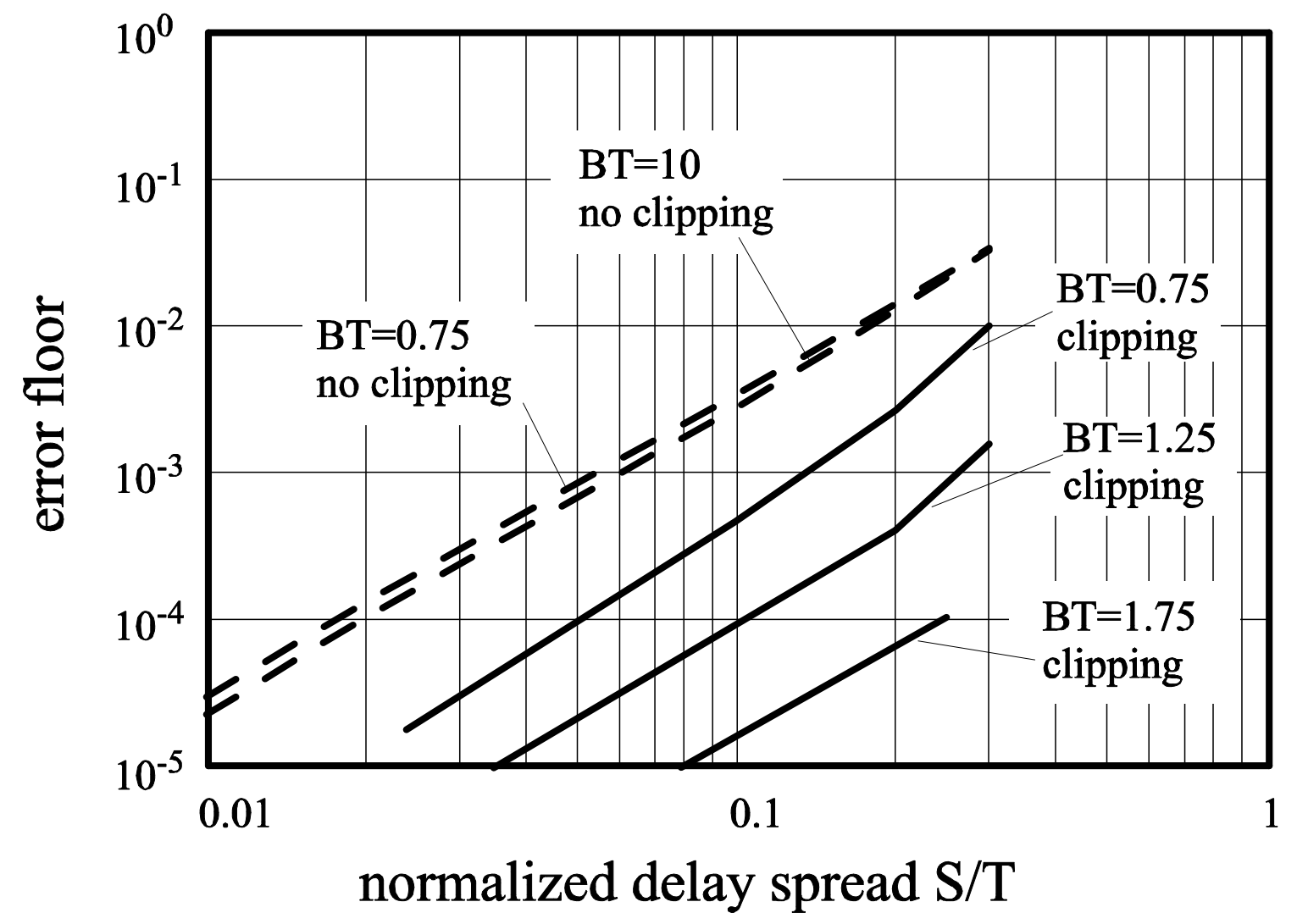

Molisch and Petrovic, Fig. 4 


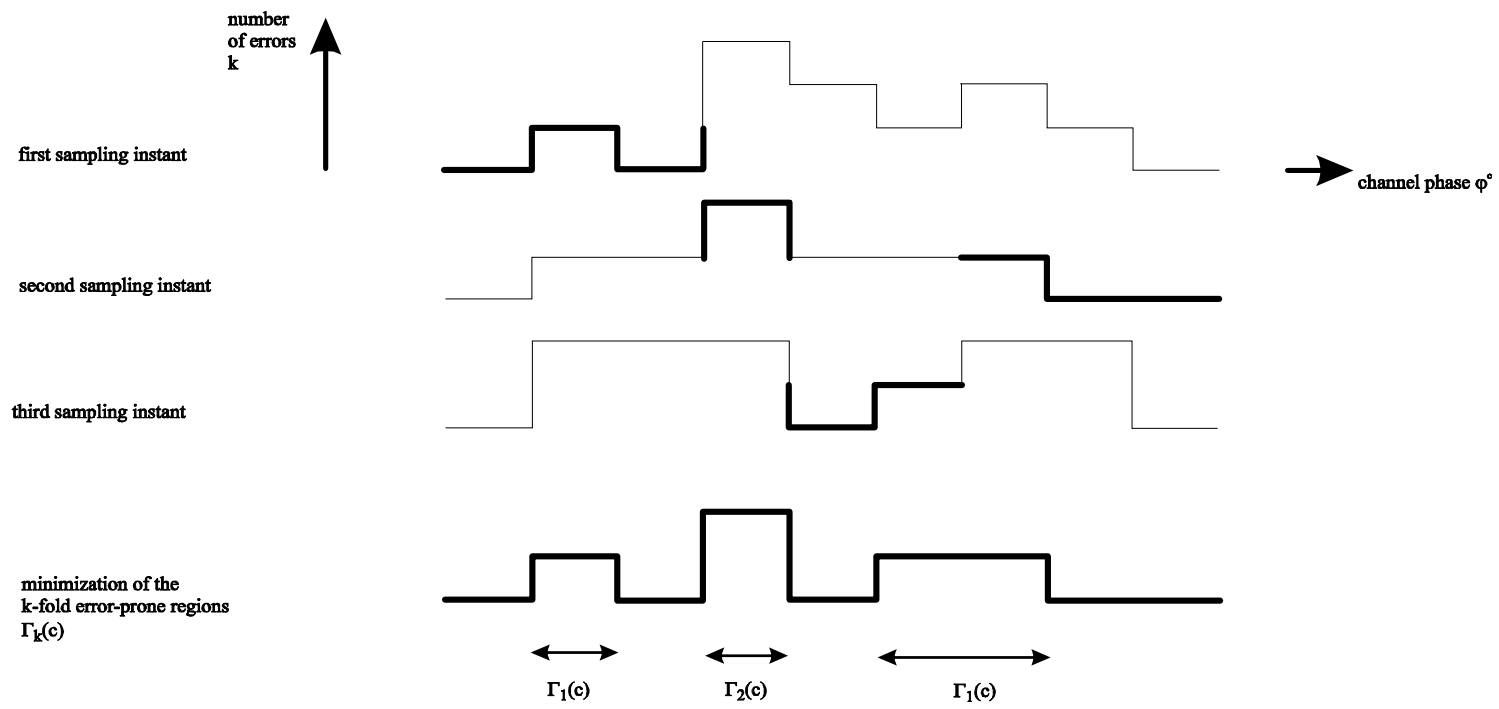

Molisch and Petrovic, Fig. 5

Fig. 5. 


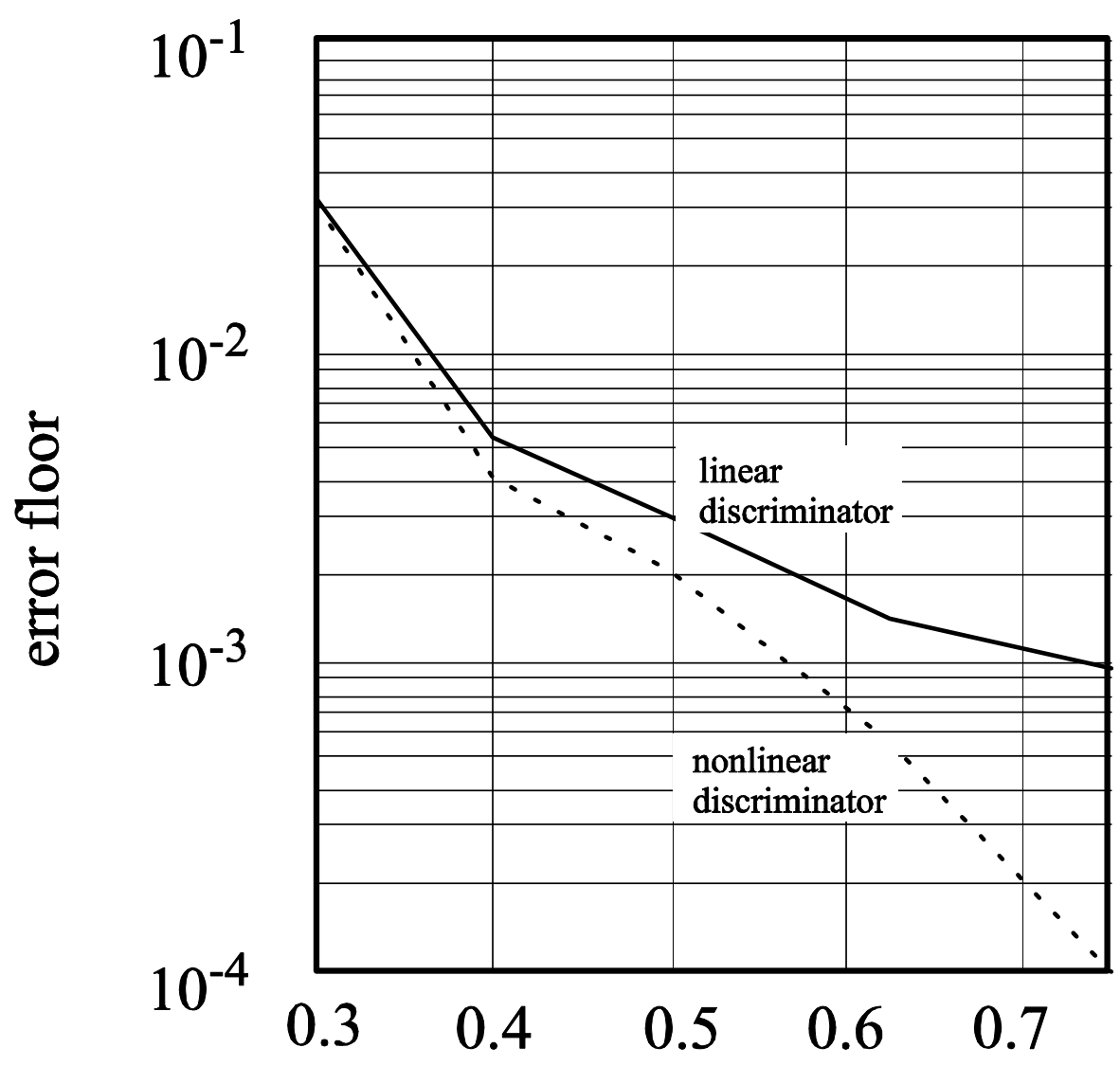

filter bandwidth

Molisch and Petrovic, Fig. 6

Fig. 6. 


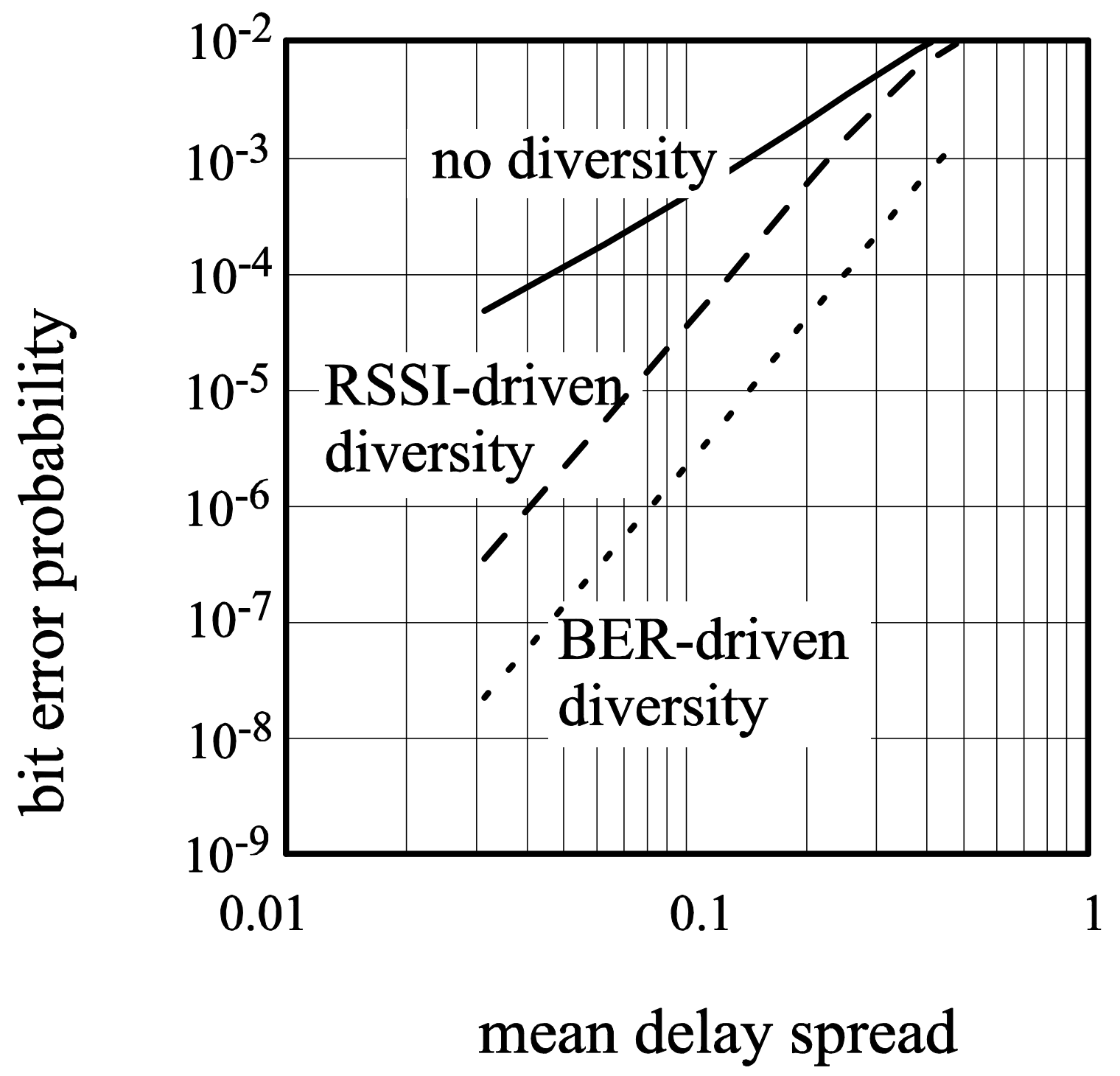

Molisch and Petrovic, Fig. 7 


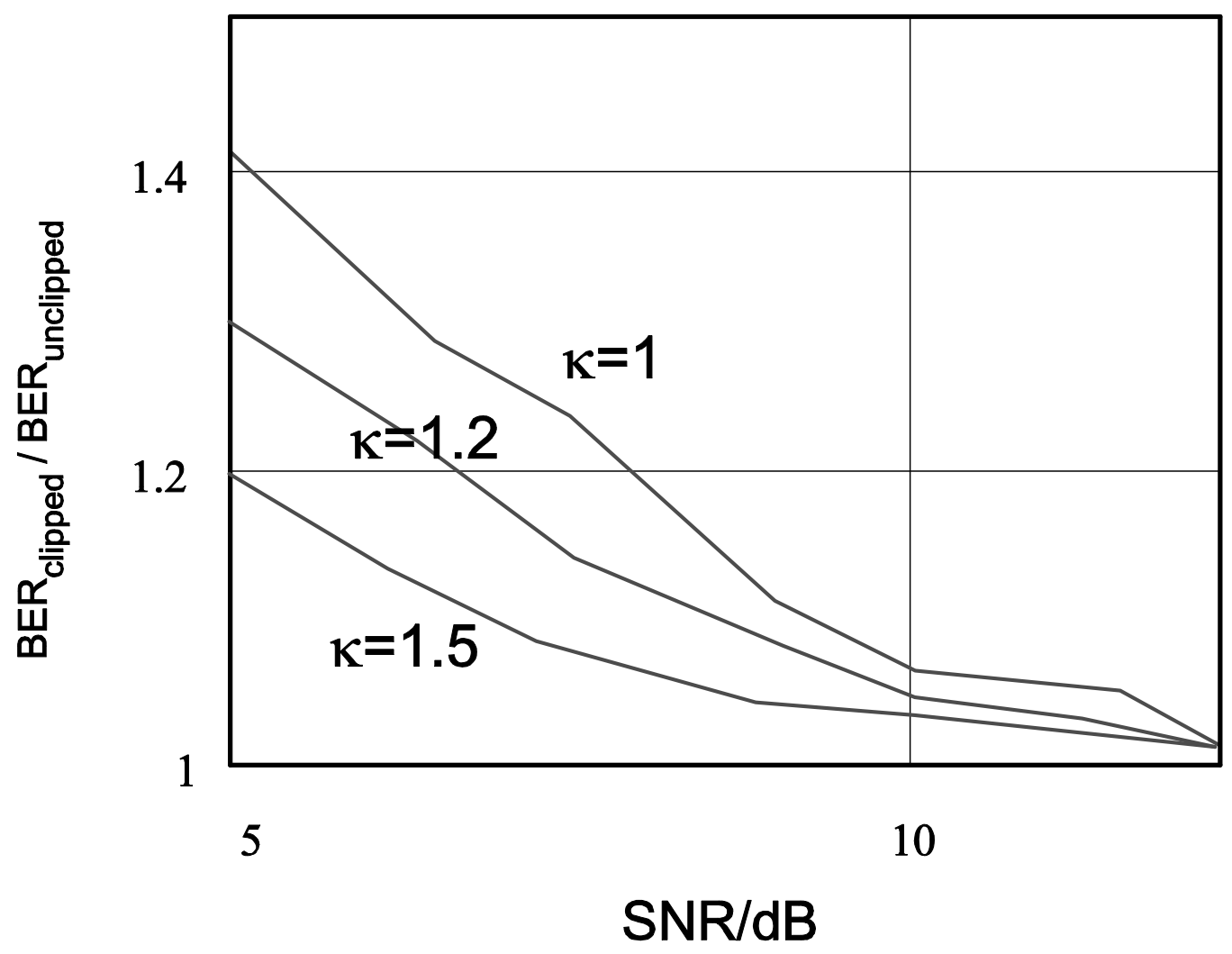

Molisch and Petrovic, Fig. 8

Fig. 8. 